\title{
A Global Route Planning Method for Intelligent Vehicles
}

\author{
Jia Xin \\ State Key Lab of Automobile \\ Simulation and Control \\ Jilin University \\ Changchun, China \\ e-mail: jia.xin@ascl.jlu.edu.cn
}

\author{
Li Kedi \\ State Key Lab of Automobile \\ Simulation and Control \\ Jilin University \\ Changchun, China \\ e-mail: li.kedi@ascl.jlu.edu.cn
}

\author{
Guan Hsin \\ State Key Lab of Automobile \\ Simulation and Control \\ Jilin University \\ Changchun, China \\ e-mail: guan.hsin@ascl.jlu.edu.cn
}

\begin{abstract}
A global route planning method for intelligent vehicle is presented here based on the comparison, selection and development of classic figure search algorithms. In addition, the effects of traffic signs in road network are also be considered in the method, so to insure that any traffic laws such as steering or passing through is violated. With the testing results, the method in the paper can decide an effective(or shortest distance) and legitimate global route for intelligent vehicle's driving guide, and the algorithm is running efficiently enough to meet the real time need when it should work temporarily during vehicle driving.
\end{abstract}

Keywords-component; Intelligent Vehicle; Global Route Planning; A* Algorithm; graph search algorithm; Traffic sign

\section{INTRODUCTION}

Global route planning is a key module in the navigation system, which is widely considered to be a fundamental problem in the field of vehicle navigation. With the development of intelligent transportation and intelligent vehicle, in order to automatically navigate driverless car, improvements on the traditional route planning methods are needed.

It can be said that if the local track decision is that a safe, legitimate and efficient track of intelligent car in the next control period is decided, based on the current road traffic environment and the state of motion, the global route planning is to plan the optimal route between the starting point and finishing point based on the abstraction road network topology, as the goal of the shortest distance or shortest driving time [1]. The local track decision is a real time work during vehicle driving, and the global route planning is generally done when the travel task has been defined.

The essence of the global route planning method for intelligent vehicle is a search algorithm in figure theory, but it has to rely on the road network expression [2], but also to meet the demand of intelligent and automotive control decision-making system. In this paper, based on the above three points, a global route planning method for intelligent vehicle is presented, and to explore the method of guiding intelligent vehicle with global route.

\section{Heuristic Search Strategy - A* Algorithm}

Figure is a class data structure in computer science, and related algorithms are basic algorithms in computer science.
There are many computational problems are defined in figure. Road network topology can also be abstracted into the figure consisting of the "nodes" and "arcs", so the problems in global route planning for intelligent vehicle can be solved with figure algorithms.

In figure related algorithms, according to some fixed sequencing, it is belong to blindness searching method that to expand the nodes in proper order or random, or namely as uninformed guiding search strategy. If the available information of the problem space is considered, to determine the sequence of nodes dynamically, and to select suitable nodes prior to expand, that is named as heuristic search strategy or informed guiding search strategy.

The easiest way to achieve heuristic search strategy is hill-climbing method (Pearl 1984) or dynamic programming method (Bellman 1956), shortly named as DP. Both of them have some limitations, which is to fall into local optimal solution easily. So it is need to find more flexible algorithm for heuristic search strategy, and the following algorithm can provide this flexibility.

People always want to be able to give priority of expansion to the most hopeful node which will lead to the destination point. A common method is to define an evaluation function to evaluate each node [3]. The evaluation function is defined as follows: $(n)=g(n)+h(n)$. Where $n$ is the index of the node being evaluated, $g(n)$ is the length of actual path from the original point to the node $n$, and $h(n)$ is the heuristic function which can give an heuristic estimation of the distance between the node $\mathrm{n}$ to the destination point. The value of $h(n)$ will guide the search to the more promising state, while the value of $g(n)$ can prevent the search developing infinitely along with the useless path. This method is called as A algorithm.

Definition $g^{*}(n)$ represents the distance of the shortest path between original point and node $\mathbf{n}$, and $k^{*}(n)$ represents the length of the actual shortest path between node $n$ and the destination point. So $f^{*}(n)$, which is $f^{*}(n)=g^{*}(n)+h^{*}(n)$, can represent the length of the shortest path from original point to destination point passing node $n$. If the heuristic function in A algorithm can satisfy $\mathrm{h}(\mathrm{n}) \leq h^{*}(n)$, the algorithm above is called as $\mathrm{A}^{*}$ algorithm [3-6].

When the search problem is solvable, $\mathrm{A}^{*}$ algorithm must be able to find an optimal solution. The time or space complexity of $\mathrm{A}^{*}$ algorithm relate to the selected heuristic function. The worst situation is equivalent to the Dijkstra 
algorithm for calculating the shortest path of a single-pair of nodes, and in other situations $A^{*}$ algorithm is better than Dijkstra algorithm [7,8].

\section{Route PlanNing Methods FOR INTELLIGENT VEHICLE BASED ON A * AlgORITHM}

In the first step work of route planning based on the $\mathrm{A}^{*}$ algorithm, the "feature points" which represent the shape of the roads are not looked as "nodes", and the relationships of these "feature points" are not looked as "edges" in the searching figure, too. It is because that with the road network information representation and format which corrects to lane, all lanes in the same road section have a potential relationship that the vehicle can change lane between every two of them, but the "feature points" on these lane have no fact connection relationship with each other in road network. So if the "feature points" and the "feature lines" in the lowest level in road network are used as "nodes" and "edges" in A* algorithm, it is not only that there will be a huge calculating workload, but also the result of search working is not the optimal route, even the effective result could not be found, since the lack of connectivity of points above.

The way to solve this problem is to restore the natural connectivity relationship between lanes including in the road sections or regions data structures when global route planning work is being done. When the $\mathrm{A}^{*}$ algorithm is used to work, the "feature facets" in road network, which are road sections or regions, looked as "nodes" in searching figure, and the connection relationships of "feature facets" are looked as "edges". This idea is similar to the bipartite graph which is used to express traffic restriction policy [9]. When a "feature facet" is found with $\mathrm{A}^{*}$ algorithm searching work, all connection relationship from it should be expanded to, which is named as "out edges". Then the free passage rules in the road sections and the region will be reacted.

If the "feature facet" also includes some traffic signs which show the information for limited accessing limitation or steering restriction, it should be considered how to filter the connections which will violate the traffic regulations when expanding its descendants. For example, to solve the problem of steering restrictions, here a judgment method with the included angle between the directions of lanes of a connection relation is presented. As shown in Figure 1, the first step is to calculate the direction of the last lane going into the connection and the direction of the lanes that the connection connects to, and the second step is to calculation the included angle of these two directions. And Then the connection relationship is classified as "going straight", "turning left", "turning right" or "U-turn". So the illegal descendants can be deleted according to traffic signs.

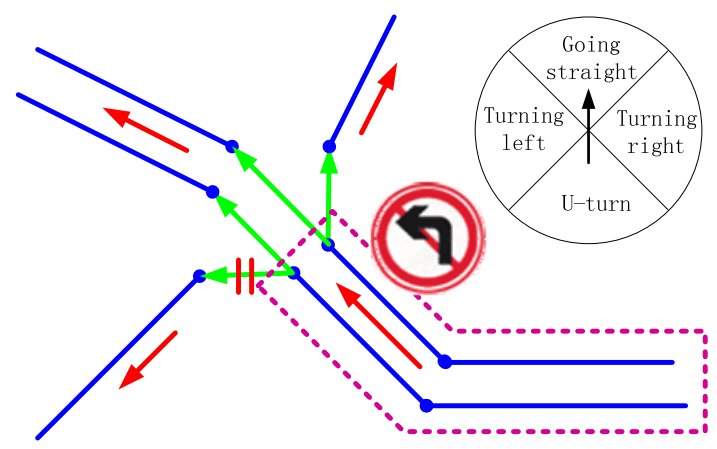

Figure 1. Method to solve traffic signs in global routing planning method

The road network searching work based on $\mathrm{A}^{*}$ algorithm finishes when the road section or region which the destination point of travel task belongs to is found. Then a sequence which consists of all the passed through road sections and regions from original point to destination point defined in travel task and the connection relationships between them. The algorithm in this level is shown in Figure 2 , in which the key steps of $A^{*}$ algorithm and how to judge legal or not of steering or passing through are shown in blocks with shadow.

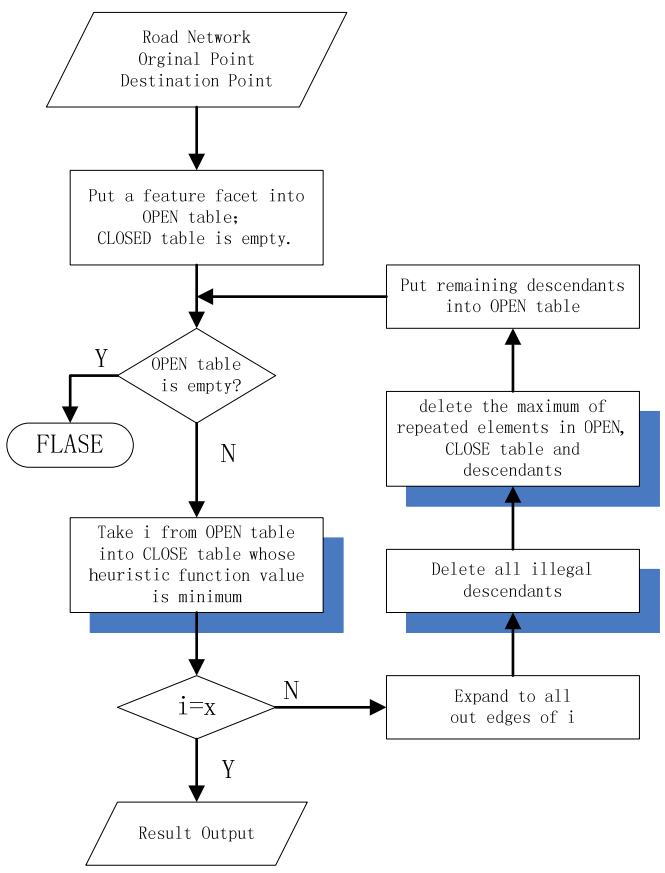

Figure 2. Flow chat of global route planning method

\section{TESTING AND ANALYZING}

\section{A. Performance Testing of A* Algorithm}

Here an example that is simplified Romania intercity road map (Figure 3) is presented. The original point is city Arad, and the destination point is city Bucharest in this 
calculation example, so that it become to "a shortest path problem with a single-pair nodes". Therefore, all kinds of blindness searching methods are suitable to the problem which can solve the shortest path problem from single source or pairs of nodes. And in this map of Romania, heuristic information can be obtained with the destination point position. It is can be proved that $h_{S L D} \leq h^{*}$ because that straight line is shortest between two points, where $h_{S L D}$ is heuristic function with straight line. So, $\mathrm{A}^{*}$ algorithm is also suitable to the problem.

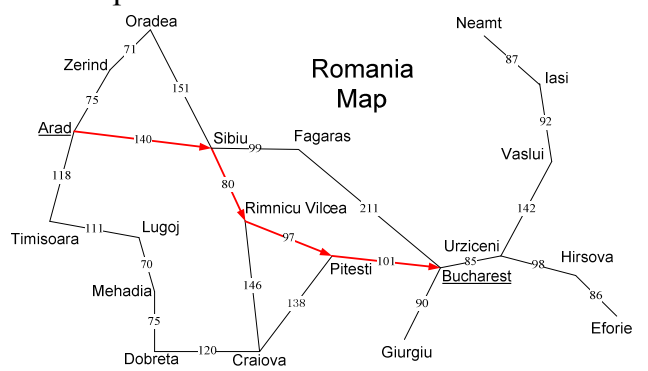

Figure 3. Testing and analyzing example for searching methods

In analysis of the performance of algorithms, focused on the following four aspects [6]: a) completeness: when the problem is solvable, this algorithm can be proved to find a solution or not; b) optimality: the optimal solution can be found with the searching strategy or not; c) time complexity: to how long it spends to find a solution; d) space complexity: how much calculating memory it consumes in the searching process. (The red route in Figure 3 is the optimal route.)

TABLE I. COMPARISON OF CLASSIC SEARCHING ALGORITHMS

\begin{tabular}{|c|c|c|c|c|c|}
\hline \multicolumn{2}{|r|}{$\begin{array}{c}\text { Searching } \\
\text { Algorithms }\end{array}$} & Optimality & Steps & Time & Memory \\
\hline \multirow{6}{*}{ 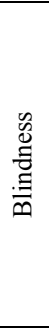 } & Breadth-prior & & $9 \sim 12$ & $\begin{array}{l}0.028 \sim \\
0.035\end{array}$ & 12 \\
\hline & Depth-prior & & $4 \sim 12$ & $\begin{array}{l}0.02 \sim \\
0.035\end{array}$ & $8 \sim 12$ \\
\hline & Bellman-Ford & $\bullet$ & 20 & 0.029 & 20 \\
\hline & Dijkstra & $\bullet$ & 20 & 0.023 & 20 \\
\hline & $\begin{array}{l}\text { Floyd- } \\
\text { Warshall }\end{array}$ & $\bullet$ & 20 & 0.024 & 400 \\
\hline & Johnson & $\bullet$ & 41 & 0.085 & 441 \\
\hline \multirow{2}{*}{ 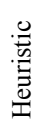 } & $\begin{array}{l}\text { Optimum- } \\
\text { prior }\end{array}$ & & 4 & 0.026 & 7 \\
\hline & $A^{*}$ & $\bullet$ & 6 & 0.033 & 10 \\
\hline
\end{tabular}

It can be seen that the $\mathrm{A}^{*}$ algorithm is some superior to others in the searching steps and search space, and can ensure the completeness and optimality of the searching result. According to Table 3, compared to blindness searching algorithms, $A^{*}$ has not much superiority in time efficiency, because there is additional expense to process heuristics, which will be made up with the superiority of searching steps in the more complex examples.

In 2007 DARPA Urban Challenge, many teams select A* algorithm or $\mathrm{A}^{*}$ developed algorithm as the foundation of the intelligent vehicle route planning method, except
Princeton University team, which are including the third one, VictorTango Team, and the forth one, Team of Massachusetts Institute of Technology (MIT) [10, 11].

The above fully explained the superiority of $\mathrm{A}^{*}$ algorithm in intelligent vehicle navigation.

\section{B. Performance Testing of Algorithm in This Paper}

Here the testing Route Network Definition File (RNDF) is presented as the example for testing which is made known before the second session of "Future Challenge" Intelligent Vehicle Competition, 2010, China (FC2010). The aerial photograph of RNDF is shown in Figure 4, in which round dots are the positions of road points, and square dots are the positions of traffic signs. Although region covered with RNDF is not big, the structure of road network is some complex, and fit to test of optimality of global route planning method. And traffic signs are is involved into RNDF in FC2010, so that it can be used to prove the legality of the searching result with the method in the paper. The fugure at upper left corner in Figure 4 is shown the road network graph, in which red round dots are the positions of traffic signs.

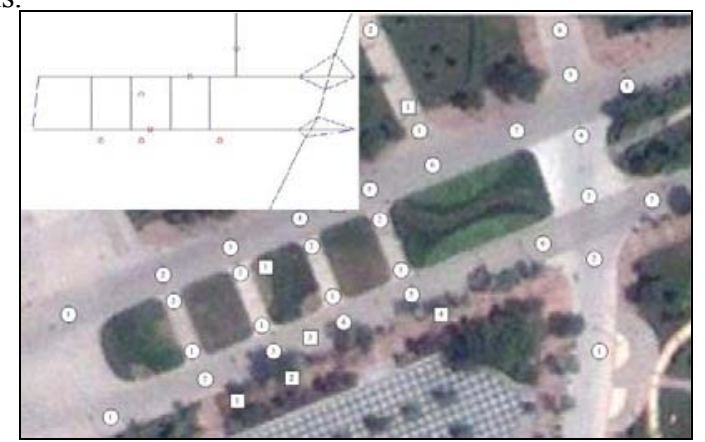

Figure 4. Testing figure of FC2010 RNDF

\section{a) Testing of Optimality of Searching Result}

In this example, the travel task is defined as that the original point is the road point (ID: 1.1.1) at lower left corner at road network graph, and the destination point is the road point (ID: 8.1.6) at upper right corner. The road network is including 31 nodes and 36 connection relationships between 8 continuous two-way-travelling lanes. The goal of method in the paper is to find the shortest path, so that the weight of every lane is its length. The heuristic function $h_{S L D}$ is straight line distance between node and destination point in searching as introduced above. In $\mathrm{C}$ code programming, "nodes" are 8 road sections which 8 lane belong to, and "edges" are 36 relationships between them. And then after 18 steps of searching work, which spent $0.124 \mathrm{~s}$, the optimal global route is planned as shown in Figure 5. In Figure 5, the red part is the searching space, and the green part is the result route. Comparing with other algorithms, such as Dijkstra algorithm, after 31 searching steps, which spent $0.187 \mathrm{~s}$, the result route is achieved just the same as the result of method above. 


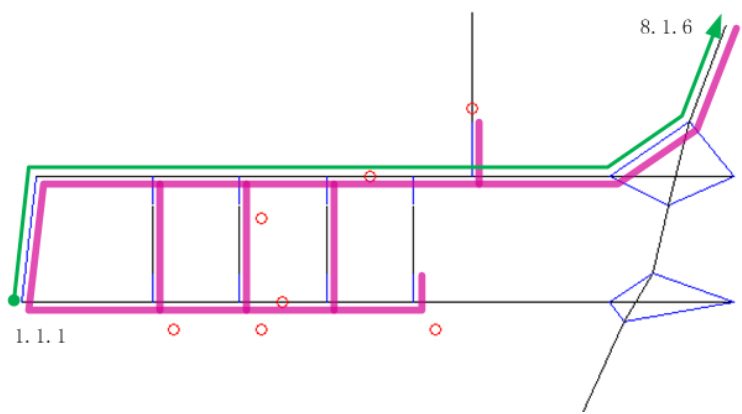

Figure 5. The searching space and the shortest path

\section{b) Testing of Legality of Searching Result}

In the test, a given traffic sign is set at every traffic sign position in FC2010 RNDF. Especially try to test the effect to global route planning result above with traffic signs, some special traffic signs which can block the road sections are set at the positions included in that result route. The project of traffic signs setting is shown in Figure 6.

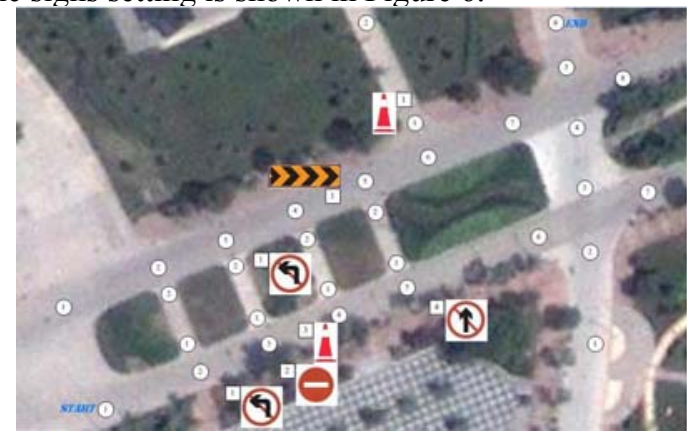

Figure 6. Testing figure of RNDF with traffic signs

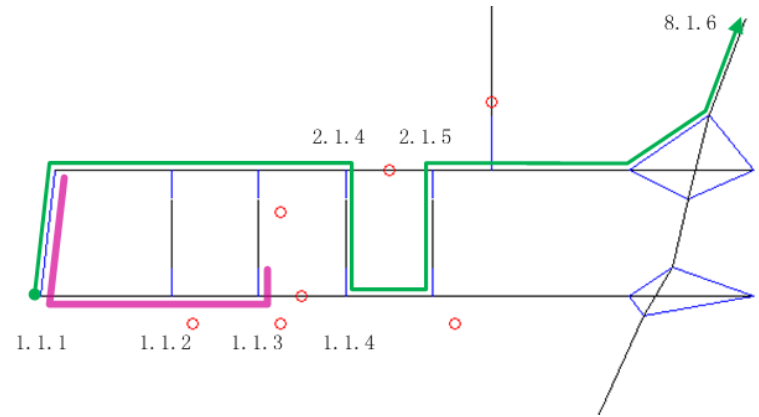

Figure 7. Searching result of legal shortest path

The result of testing is that after 23 steps of searching work, which spent $0.140 \mathrm{~s}$, the result route is achieved as shown in Figure 7, the green part. According with the project of traffic signs setting shown in Figure 6, the result route is legal for all the accessing limitation and steering restriction in road network and it also a shortest path. At the same time, it is proved that the traffic sign information in RNDF able to play its due role to provide complete information for global route planning.

\section{CONCLUSION}

With the comparison of the classic path planning algorithm, and to meet the algorithm running efficiency and accuracy requirements, the $\mathrm{A}^{*}$ algorithm is chose to be the theoretical basis of global route planning method for intelligent vehicle. And considering the restrictions with traffic signs to the steering and passing through, the shortest route planning method is raised, which is that define "node" as road sections and zone segments, and define "edge" as the relationship between them, and the legality is decided with included angle with lanes. Programming test with test intelligent vehicle, then the optimality and legality of this global route planning method is tested with comparison to classic figure search algorithms running results, and the improved algorithm performance advantages is reflected.

\section{ACKNOWLEDGMENT}

The study in the paper is supported with Program for Changjiang Scholars and Innovative Research Team in University (No. IRT0626).

\section{REFERENCES}

[1] Martin Buehler, Karl Iagnemma, Sanjiv Singh. The DARPA Urban Challenge, Springer, 2010.

[2] Li Kedi. "Research on Road Network Expression \& Global Route Planning for Intelligent Vehicles", Master Dissertation, Jilin University, 2011.

[3] George F. Luger. "Artificial Intelligence : Structures and Strategies for Complex Problem Solving”, China Machine Press, 2005.

[4] Hart E.P, Nilsson N.J, Raphael B. "A Formal Basis for the Heuristic Determination of Minimum Cost Paths", IEEE Trans, Syst.Sci.Cybern., 1968, vol. 4, no. 2.

[5] Hart E P,Nilsson N J,Raphael B. "Correction to A Formal Basis for the Heuristic Determination of Minimum Cost Paths", SIGART Newsletter, 1972, vol. 37.

[6] Stuart J. Russell, Peter Norvig. "Artificial Intelligence : A Modern Approach", Tsinghua University Press, 2006.

[7] Dijkstra E.W. "A Note on Two Problems in Connexion with Graphs “" Numerische Mathematik, 1959, vol. 1.

[8] Cormen, T.H.. "Introduction to Algorithms", China Machine Press, Beijing, China, 2006.

[9] Gao Peng. "Research on the constrained K-shortest paths algorithm within a dynamic restricted searching area ", Master Dissertation, Jilin University, 2009.

[10] Charles Reinholtz, etc. DARPA Urban Challenge Technical Paper, 2007.

[11] John Leonard, etc. Team MIT Urban Challenge Technical Report, 2007 